mit dem „aktiven“ Placebo beobachtet werden. Damit verbunden war eine gesteigerte $\mu$-Opioidrezeptor-vermittelte Neurotransmission im subgenualen anterioren Cingulum, im Nucleus accumbens, im Thalamus und in der Amygdala, alles Regionen, denen eine pathophysiologische Bedeutung hinsichtlich depressiver Symptome zugesprochen wird.

\section{Kommentar}

Die hier vorliegenden Daten veranschaulichen, welchen Effekt die Überzeugung eines Patienten hinsichtlich der Wirksamkeit einer Behandlung auf die tatsächliche Wirksamkeit haben kann. Für den klinischen Alltag wird nochmals unterstrichen, wie wichtig die Interaktion zwischen Behandler und Patient hinsichtlich der Wirksamkeit einer Therapie ist. Als Ausblick könnte eine expe- rimentelle Beeinflussung der Opioidrezeptorsystems zukünftig ein weiterer Ansatzpunkt für das bessere Verständnis der Neurobiochemie affektiver Störungen werden.

Dr. Christian Eberlein

Peciña M, Bohnert AS, Sikora M et al. Association between placebo-activated neural systems and antidepressant responses: neurochemistry of placebo effects in major depression. JAMA

Psychiatry 2015; 72 (11): 1087-94

\title{
Auf der Suche nach dem Depressionsschalter
}

\section{Intraoperative Untersuchungen während der Implantation von Elektroden zur tiefen Hirnstimulation (THS) öffnen auf eine einzigartige Weise ein Fenster zum Verständnis der Lokalisationen verschiedener Hirnfunktionen. Eine Studie zeigt, dass es auch für affektive Veränderungen spezifische Ziel- regionen zu geben scheint.}

$B^{c}$ ei der Behandlung von Bewegungsstörungen durch THS können seit Jahren intraoperativ die Wirksamkeit der angestrebten Behandlung überprüft und unerwünschte motorische Effekte reduziert werden. Bei der noch nicht so gut untersuchten Behandlung affektiver Störungen stellt sich vielmehr die Frage nach akuten Gefühlsänderungen. $\mathrm{Ob}$ es einen „Schalter“ für die Veränderung des Affektes gibt, sollte in der vorliegenden Studie untersucht werden.

Das Team der Emory University School of Medicine aus Atlanta untersuchte eine kleine Gruppe von neun $\mathrm{Pa}$ tienten mit therapieresistenter Depression, die mittels THS behandelt wurden.
In jeder Hemisphäre wurden Elektroden mit je vier Kontakten in den subgenualen Gyrus cinguli implantiert und die Reaktionen der Patienten auf die Stimulation erfasst. Es konnten Veränderungen in der extero- und interozeptiven Wahrnehmung identifiziert werden. Die Untersuchung wurde doppelt verblindet, es wurden 72 tatsächliche Stimulationen von drei Minuten Dauer durchgeführt und 36 Sham-Stimulationen.

Bei den Verumstimulationen kam es im Mittel bei 30 von 72 Stimulationen $\mathrm{zu}$ einer transienten Verbesserung der interozeptiven Wahrnehmung, was nur bei vier von 36 Sham-Stimulationen der Fall war. $\mathrm{Zu}$ einer Veränderung der extero- zeptiven Wahrnehmung kam es im Mittel bei neun der 72 Verumstimulationen aber bei keiner der Sham-Stimulationen. Mittels probabilistischer Traktografie wurden Faserbündelverbindungen zum ventromedialen frontalen Kortex und zum anterioren Cingulum identifiziert, die bei der hier gewählten Form der Stimulation eine Rolle zu spielen scheinen.

\section{Kommentar}

Auch für affektive Veränderungen scheint es spezifische Zielregionen zu geben, die mittels THS therapeutisch genutzt werden können. Auch wenn es sich hier nur um eine kleine Anzahl von Patienten handelt, ermutigen die Ergebnisse doch zu weiteren Studien in diesem Bereich, wobei vor allem Längsschnittbeobachtungen von Interesse sein werden.

Dr. Christian Eberlein

Choi KS, Riva-Posse P, Gross RE et al. Mapping the "depression switch" during intraoperative testing of subcallosal cingulate deep brain stimulation. JAMA Neurol 2015; 72 (11): $1252-60$

\section{Antidepressiva - verbesserte Wirkung durch „measurement-based care"?}

\begin{abstract}
"Measurement-based care" bedeutet, dass neben der Anwendung eines Therapiealgorithmus durch regelmäßige Überprüfung psychiatrisch relevanter Symptome und unerwünschter Arzneimittelwirkungen mittels Fragebögen eine individuelle Anpassung der antidepressiven Therapie möglich wird.
\end{abstract}

D ie GAP-Studien und die STAR ${ }^{\star} \mathrm{D}$ Studie haben erste Hinweise geliefert, dass durch „measurement-based care" eine suffizientere Therapie mit besserem Outcome ermöglicht werden kann. Die Arbeitsgruppe um Ton Guo befasste sich nun mit der Frage, ob das Prinzip der "measurement-based care“ bei der medikamentösen Behandlung depressiver Patienten im Vergleich zur regulären Behandlung zu einem schnelleren Ansprechen und zu einer schnelleren Remission führt. Hierfür wurden insgesamt 120 ambulante Patienten mit einer mittelgradigen bis schweren depressiven Symptomatik über einen Zeitraum von 24 Wochen fortlaufend randomisiert in je eine Studien- oder Kontrollgruppe eingeteilt. Die antidepressive Medikation war in beiden Gruppen auf Paroxetin und Mirtazapin beschränkt. Depressive Symptome wurden in der Studiengruppe mithilfe des HAM-D (Hamilton Depression Rating Scale) und des QIDS-SR (Quick Inventory of De- 